Proceedings of the Edinburgh Mathematical Society (2003) 46, 221-227 (C)

DOI:10.1017/S0013091501000578 Printed in the United Kingdom

\title{
A COUNTEREXAMPLE TO METRIC DIFFERENTIABILITY
}

\author{
BERND KIRCHHEIM $^{1}$ AND VALENTINO MAGNANI ${ }^{2}$ \\ ${ }^{1}$ Max-Plank-Institut für Mathematik in den Naturwissenschaften, \\ Inselstrasse 22-26, 04103 Leipzig, Germany (kirchheim@mis.mpg.de) \\ ${ }^{2}$ Scuola Normale Superiore, Piazza dei Cavalieri \%, \\ 56126 Pisa, Italy (magnani@cibs.sns.it)
}

(Received 31 May 2001)

\begin{abstract}
We show that the metric version of Pansu's differentiability result for Lipschitz maps fails; this illustrates an interesting difference between Euclidean domains and domains that are non-abelian stratified groups.
\end{abstract}

Keywords: metric differentiability; nilpotent groups

AMS 2000 Mathematics subject classification: Primary 26B05

Secondary 22E25

\section{Introduction}

Differentiability of Lipschitz maps is a basic tool for tackling several questions in geometric measure theory. In fact, Lipschitz maps allow a natural generalization of the notion of surface. In a general metric space, these are the subsets which are parametrized by Lipschitz maps defined on some Euclidean space. These subsets are called rectifiable. When the target is another Euclidean space, by Rademacher's differentiability theorem, many of the classical properties of smooth surfaces can be extended to rectifiable sets (see [4] or [14] for a complete presentation of the subject). Recently, some properties of rectifiable sets as the existence almost everywhere of tangent spaces, the regularity of their Hausdorff measure, area and co-area formulae have been extended to general metric spaces (see $[\mathbf{1}, \mathbf{9}, \mathbf{1 0}]$ ). The key idea is to replace the notion of differentiability with a weaker one, namely metric differentiability, and to prove that any metric-valued Lipschitz map defined on a Euclidean space is metrically differentiable.

In $[\mathbf{1 5}]$, on the other hand, Rademacher's theorem was generalized from the Euclidean setting to the framework of non-abelian stratified groups. This boosted the development of geometric measure theory methods in such groups (see, for example, $[\mathbf{2}, \mathbf{6}, \mathbf{7}, \mathbf{1 1}-\mathbf{1 3}$, 16-18]).

In [16], a further extension of the metric differentiability into this non-abelian framework is achieved and used as the main tool to obtain the non-existence of quasi-isometric 
embeddings of non-abelian stratified groups into Alexandrov metric spaces with nonnegative or non-positive curvature in the sense of Topanogov. More precisely, in [16] a partial metric differentiability of Lipschitz maps along the so-called horizontal directions of the group is proved, leaving open the question of complete metric differentiability, as posed in the same paper in Remark 3.

The question of whether the full metric version of Pansu's extension of Rademacher's theorem is valid also arises in another context. In $[\mathbf{3}, \S 11.4]$, David and Semmes note that metric differentiability is perhaps the most powerful tool for finding bi-Lipschitz pieces of mappings and for deciding which metric spaces look down on others. In [8, Question 22], Heinonen and Semmes asked in particular whether the three-dimensional Heisenberg group looks down on all other spaces. Of course, this would be an easy consequence of the metric differentiability.

In this note, however, we present a counterexample showing that the metric differentiability of Lipschitz maps may fail when the domain of the map is a non-abelian stratified group, instead of a Euclidean space.

\section{Some basic definitions}

In this brief section we recall the main notions we are going to use. A stratified group is a graded, nilpotent, simply connected Lie group $\mathbb{G}$, such that there exists a subspace of left-invariant vector fields which generate all of the Lie algebra $\mathcal{G}$ with respect to the Lie product of vector fields. We have a grading $\mathcal{G}=V_{1} \oplus \cdots \oplus V_{n}$ and a one-parameter group of dilations. Setting $\delta_{r}: \mathcal{G} \rightarrow \mathcal{G}$,

$$
\delta_{r}\left(\sum_{i=1}^{n} v_{i}\right)=\sum_{i=1}^{n} r^{i} v_{i}, \quad r>0,
$$

where $v_{i} \in V_{i}$, for $i=1, \ldots, n$. The integer $n$ is called the degree of nilpotency of the group (see [5] for more information on stratified groups). These types of groups can be endowed with a natural left-invariant distance $d: \mathbb{G} \times \mathbb{G} \rightarrow[0,+\infty[$ which is homogeneous with respect to the group of self-similarities, that is

$$
d\left(\delta_{r} x, \delta_{r} y\right)=r d(x, y)
$$

for any $r>0, x, y \in \mathbb{G}$. Note that we have identified the group with its Lie algebra, using the fact that $\mathbb{G}$ is simply connected. Hence there exists a diffeomorphism between $\mathbb{G}$ and $\mathcal{G}$.

Now we introduce the definition of metric differentiability generalized to stratified groups.

Definition 2.1. We say that a map $\nu: \mathbb{G} \rightarrow[0,+\infty[$ is a homogeneous seminorm if for each $x, y \in \mathbb{G}$ and $r>0$ we have

(1) $\nu\left(\delta_{r} x\right)=r \nu(x)$

(2) $\nu(x y) \leqslant \nu(x)+\nu(y)$. 
Definition 2.2. Let $(Y, \rho)$ and $(\mathbb{G}, d)$ be a metric space and a stratified group, respectively. We say that a map $f: A \rightarrow Y$, where $A$ is an open subset of $\mathbb{G}$, is metrically differentiable at $x \in A$, if there exists a homogeneous seminorm $\nu_{x}$ such that

$$
\frac{\rho\left(f\left(x \delta_{t} v\right), f(x)\right)}{t} \rightarrow \nu_{x}(v), \quad \text { as } t \rightarrow 0^{+},
$$

uniformly in $v$ which varies in a compact neighbourhood of the unit element.

Remark 2.3. We point out that if $\mathbb{G}=\mathbb{R}^{n}$, then any Lipschitz map is metrically differentiable almost everywhere, as proved in [1], [9] and [10]. Furthermore, in $[\mathbf{1 6}]$ it is shown that bi-Lipschitz maps are almost everywhere metric differentiable on stratified groups if one allows the direction $v$ to vary only among the elements of $V_{1}$, namely the horizontal directions. The latter result directly applies also to an arbitrary Lipschitz map $f: \mathbb{G} \rightarrow Y$, since it follows from the metric differentiability of the bi-Lipschitz graph map $(x \rightarrow(x, f(x)))$ into the $\ell_{1}$-product $\mathbb{G} \times Y$. So, it is clear that we will consider non-horizontal directions in order to show that the metric differentiability does not hold in general.

The stratified group we choose to build our counterexample is the three-dimensional Heisenberg group $\mathbb{H}$, which can be linearly identified with $\mathbb{R}^{3}$. We denote the elements $\eta, \xi \in \mathbb{H}$ as $\xi=(z, t), \eta=(w, \tau)$, where $z=\left(z_{1}, z_{2}\right), w=\left(w_{1}, w_{2}\right)$ belong to $\mathbb{R}^{2}$. As usual, on $\mathbb{H}$ we have the non-abelian group operation

$$
(z, t)(w, \tau)=\left(z+w, t+\tau+2\left(z_{1} w_{2}-z_{2} w_{1}\right)\right) .
$$

In this case the non-horizontal directions are of the type $(0,0, s)$, with $s \neq 0$. We consider $G: \mathbb{H} \rightarrow \mathbb{R}$, defined as $G(z, t)=|z| \vee \sqrt{|t|}$, where the symbol ' $\vee$ ' denotes the 'maximum' operation. It is known that $d(\xi, \eta)=G\left(\xi^{-1} \eta\right)$, for $\xi, \eta \in \mathbb{H}$, yields a left-invariant distance in the Heisenberg group (see, for example, $[6]$ ). The dilations $\delta_{r}: \mathbb{H} \rightarrow \mathbb{H}$ are defined as $\delta_{r}((z, t))=\left(r z, r^{2} t\right)$. It is clear that these dilations scale homogeneously with the distance $d$.

\section{The counterexample}

In this section we build a new metric $\rho$ on $\mathbb{H}$ such that the identity map $I: \mathbb{H} \rightarrow \mathbb{H}$ is a Lipschitz function with respect to the homogeneous distance $d$ on the domain and the metric $\rho$ on the codomain, more precisely a 1-Lipschitz function. We will show that with this distance the metric differentiability fails. We have seen that a homogeneous distance in the Heisenberg group can be defined as $d(\xi, \eta)=G\left(\xi^{-1} \eta\right)$, where $G(z, t)=|z| \vee \sqrt{|t|}$. We obtain our counterexample by replacing the square root function in the definition of $G$ with a concave map $g:[0,+\infty[\rightarrow[0,+\infty[$ such that the function $S: \mathbb{H} \rightarrow \mathbb{R}$, $S(z, t)=|z| \vee g(|t|)$ satisfies the following three claims.

(1) The function $S: \mathbb{H} \rightarrow \mathbb{R}$ yields a left-invariant metric on $\mathbb{H}$ which is defined as $\rho(\xi, \eta)=S\left(\xi^{-1} \eta\right), \xi, \eta \in \mathbb{H}$.

(2) The map $I:(\mathbb{H}, d) \rightarrow(\mathbb{H}, \rho)$ is 1-Lipschitz. 
(3) If we consider the non-horizontal direction $v=(0,0,1) \in \mathbb{H}$, then for any $\zeta \in \mathbb{H}$ there does not exist the limit of

$$
\frac{\rho\left(I\left(\zeta \delta_{t} v\right), I(\zeta)\right)}{t}=\frac{\rho\left(\delta_{t} v, 0\right)}{t}, \quad \text { as } t \rightarrow 0_{+} ;
$$

in fact, we reach the maximal possible oscillation of the quotient:

$$
\limsup _{t \rightarrow 0_{+}} \frac{\rho\left(I\left(\zeta \delta_{t} v\right), I(\zeta)\right)}{t}=1, \quad \liminf _{t \rightarrow 0_{+}} \frac{\rho\left(I\left(\zeta \delta_{t} v\right), I(\zeta)\right)}{t}=0 .
$$

Claim (3) says in particular that the 1-Lipschitz map $I:(\mathbb{H}, d) \rightarrow(\mathbb{H}, \rho)$ is not metrically differentiable at any point of $\mathbb{H}$. The following two theorems will prove the existence of a map $g:[0,+\infty[\rightarrow[0,+\infty[$ such that our claims are satisfied and in this way establish the counterexample.

Theorem 3.1. Let $\kappa:[0,+\infty[\rightarrow[0,+\infty[$ be a convex, strictly increasing function which is continuous at the origin and satisfies $\kappa(0)=0$. Then, defining $h(t)=\kappa(t)+t^{2}$, the concave map $g=h^{-1}$ yields a function $S(z, t)=|z| \vee g(|t|)$ which satisfies claims (1) and (2).

Proof. The convexity and the continuity at the origin of $\kappa$ imply $\kappa(t)+\kappa(s) \leqslant \kappa(t+s)$ for any $t, s \geqslant 0$, hence

$$
h(t+s) \geqslant h(t)+h(s)+2 t s, \quad \text { for } t, s \geqslant 0 .
$$

The function $h(t)=\kappa(t)+t^{2}$ is strictly monotone, thus $g=h^{-1}$ is well defined and $S(z, t)=|z| \vee g(|t|)$ also. The triangle inequality for the function $\rho(\xi, \eta)=S\left(\xi^{-1} \eta\right)$ is equivalent to $S(\xi \eta) \leqslant S(\xi)+S(\eta)$, for every $\xi, \eta \in \mathbb{H}$. We denote $\xi=(z, t), \eta=(w, \tau)$, where $z=\left(z_{1}, z_{2}\right)$ and $w=\left(w_{1}, w_{2}\right)$, and then

$$
S(\xi \eta)=|z+w| \vee g\left(\left|t+\tau+2\left(z_{1} w_{2}-z_{2} w_{1}\right)\right|\right) .
$$

If $|z+w| \geqslant g\left(\left|t+\tau+2\left(z_{1} w_{2}-z_{2} w_{1}\right)\right|\right)$, then we clearly have

$$
S(\xi \eta)=|z+w| \leqslant|z|+|w| \leqslant S(\xi)+S(\eta) .
$$

So, our inequality holds if we prove that

$$
g\left(\left|t+\tau+2\left(z_{1} w_{2}-z_{2} w_{1}\right)\right|\right) \leqslant S(\xi)+S(\eta) .
$$

We have

$$
\left|t+\tau+2\left(z_{1} w_{2}-z_{2} w_{1}\right)\right| \leqslant|t|+|\tau|+2\left|\left(z_{1}, z_{2}\right) \cdot\left(w_{2},-w_{1}\right)\right| \leqslant|t|+|\tau|+2|z||w|
$$

and $|t|=h(g(|t|)) \leqslant h(S(\xi)),|\tau|=h(g(|\tau|)) \leqslant h(S(\eta))$, hence

$$
\left|t+\tau+2\left(z_{1} w_{2}-z_{2} w_{1}\right)\right| \leqslant h(S(\xi))+h(S(\eta))+2 S(\xi) S(\eta) .
$$

The latter inequality and property (3.1) give $\left|t+\tau+2\left(z_{1} w_{2}-z_{2} w_{1}\right)\right| \leqslant h(S(\xi)+S(\eta))$, which corresponds to $g\left(\left|t+\tau+2\left(z_{1} w_{2}-z_{2} w_{1}\right)\right|\right) \leqslant S(\xi)+S(\eta)$. It remains to prove that $I:(\mathbb{H}, d) \rightarrow(\mathbb{H}, \rho)$ is 1 -Lipschitz. This fact is equivalent to showing that $S \leqslant G$, which is true if $g(|t|) \leqslant \sqrt{t}$, that is $|t| \leqslant h(\sqrt{|t|})=\kappa(\sqrt{t})+|t|$. So the proof is complete. 
Now, among all the maps $\kappa$ which have the properties assumed in the preceding lemma, we want to find a particular one which produces the oscillation required in claim (3). We notice that if $v=(0,0,1) \in \mathbb{H}$, then $\rho\left(I\left(\zeta \delta_{t} v\right), I(\zeta)\right)=\rho\left(\delta_{t} v, 0\right)=g\left(t^{2}\right)$, so claim (3) is equivalent to requiring the following:

$$
\limsup _{t \rightarrow 0_{+}} \frac{g\left(t^{2}\right)}{t}=1, \quad \liminf _{t \rightarrow 0_{+}} \frac{g\left(t^{2}\right)}{t}=0,
$$

where $g=h^{-1}$ and $h(t)=\kappa(t)+t^{2}$.

Theorem 3.2. There exists a strictly increasing convex map $\kappa:[0,+\infty[\rightarrow[0,+\infty[$, continuous at the origin, with $\kappa(0)=0$, such that, defining $g=h^{-1}$, with $h(t)=\kappa(t)+t^{2}$, $t \geqslant 0$, the upper and lower limits as given in (3.3) hold.

Proof. It is easy to see that the requirements (3.3) for $g$ are equivalent to the conditions

$$
\limsup _{t \rightarrow 0_{+}} \frac{\kappa(t)}{t^{2}}=+\infty \quad \text { and } \quad \liminf _{t \rightarrow 0_{+}} \frac{\kappa(t)}{t^{2}}=0
$$

on the corresponding function $\kappa$. To find such a $\kappa$, we use the following simple observation. If we are given an affine, increasing function $\kappa$ that vanishes at some positive number $t^{\prime}$ very close to zero, then the quotient $\kappa(t) / t^{2}$ oscillates a lot. Indeed, if $t$ declines from 1 towards $t^{\prime}$, then the quotient first gets very large and then approaches zero. Stopping shortly before $t^{\prime}$, we can connect $\kappa$ to another affine function with smaller but still positive slope that vanishes much closer to zero. Thus, the considered quotient oscillates along the new function even more and the combined function is convex.

To make this argument precise, we fix two positive sequences $\left.\left(\varepsilon_{l}\right) \subset\right] 0,1\left[,\left(m_{l}\right) \subset\right.$ ]0, $+\infty$ [, with $\varepsilon_{l} \rightarrow 0$ and $m_{l} \rightarrow+\infty$ as $l \rightarrow \infty$. We consider an arbitrary number $b_{0}>0$ and choose $t_{0}, a_{0}>0$ such that $t_{0} \varepsilon_{0}<b_{0}, a_{0}<\varepsilon_{0} t_{0}^{2}$. Then we define $\kappa_{0}(t)=a_{0}+b_{0}\left(t-t_{0}\right)$, observing that $\kappa_{0}\left(t_{0}\right) / t_{0}^{2}<\varepsilon_{0}$. We consider $\beta_{1}=a_{0} / t_{0}<t_{0} \varepsilon_{0}<b_{0}$ and fix $\left.\tau_{1} \in\right] 0, t_{0}[$ such that $\beta_{1} / \tau_{1}>m_{1}$. We observe that

$$
\lim _{b \rightarrow \beta_{1}^{+}} \frac{b}{\tau_{1}}+\frac{\left(\beta_{1}-b\right) t_{0}}{\tau_{1}^{2}}=\frac{\beta_{1}}{\tau_{1}}>m_{1}, \quad \lim _{b \rightarrow \beta_{1}^{+}} \frac{t_{0}\left(b-\beta_{1}\right)}{b^{2}}=0 ;
$$

hence we can choose $\left.b_{1} \in\right] \beta_{1}, b_{0}[$ such that

$$
\frac{b_{1}}{\tau_{1}}+\frac{\left(\beta_{1}-b_{1}\right) t_{0}}{\tau_{1}^{2}}>m_{1} \quad \text { and } \quad \frac{t_{0}\left(b_{1}-\beta_{1}\right)}{b_{1}^{2}}<\frac{1}{2} .
$$

We now define $\kappa_{1}(t)=t_{0}\left(\beta_{1}-b_{1}\right)+b_{1} t$, so by the first inequality (3.5) we have $\kappa_{1}\left(\tau_{1}\right) / \tau_{1}^{2}>m_{1}$ and $\kappa_{1}\left(t_{0}\right)=\beta_{1} t_{0}=a_{0}=\kappa_{0}\left(t_{0}\right)$. We note that $\kappa_{1}(\bar{t})=0$ if and only if $\bar{t}=t_{0}\left(b_{1}-\beta_{1}\right) / b_{1}>0$. By the second inequality of (3.4) we get $\bar{t}<\frac{1}{2} b_{1}$, and since $\kappa_{1}\left(\tau_{1}\right)>0$ we infer that $\bar{t}<\tau_{1}$. Thus, we can choose $\left.t_{1} \in\right] \bar{t}, \min \left(\tau_{1}, \frac{1}{2} b_{1}\right)[\operatorname{such}$ that $\kappa_{1}\left(t_{1}\right)<\varepsilon_{1} t_{1}^{2}$ and $t_{1} \varepsilon_{1}<b_{1}$. Defining $a_{1}=\kappa_{1}\left(t_{1}\right)$, we see that $\kappa_{1}(t)=a_{1}+b_{1}\left(t-t_{1}\right)$ 
and we have shown that for every $b_{0}, a_{0}, t_{0}, m_{1}>0$, with $a_{0} / t_{0}<b_{0}$, for each $\varepsilon_{1}>0$ and $m_{1} \in \mathbb{R}$ there exist $t_{1}<\tau_{1}$ in $] 0, t_{0}\left[\right.$ and $\left.a_{1}>0, b_{1} \in\right] 0, b_{0}[$ such that

$$
\begin{gathered}
\kappa_{1}\left(t_{0}\right)=\kappa_{0}\left(t_{0}\right), \quad \kappa_{1}\left(\tau_{1}\right) / \tau_{1}^{2}>m_{1}, \\
\kappa_{1}\left(t_{1}\right) / t_{1}^{2}<\varepsilon_{1}, \quad \kappa_{1}\left(t_{1}\right) / t_{1}<b_{1}<b_{0}, \quad t_{1}<\frac{1}{2} b_{1} .
\end{gathered}
$$

This procedure can be iterated by induction, obtaining for each $j \geqslant 1$ that there exists $\left.\tau_{j}, t_{j}>0, \tau_{j} \in\right] t_{j}, t_{j-1}\left[\right.$, and $a_{j}, b_{j}>0$ such that the map $\kappa_{j}(t)=a_{j}+b_{j}\left(t-t_{j}\right)$ satisfies

$$
\left.\begin{array}{c}
\kappa_{j}\left(t_{j-1}\right)=\kappa_{j-1}\left(t_{j-1}\right), \quad b_{j}<b_{j-1}, \\
\kappa_{j}\left(\tau_{j}\right) / \tau_{j}^{2}>m_{j}, \quad \kappa_{j}\left(t_{j}\right) / t_{j}^{2}<\varepsilon_{j}, \quad t_{j}<2^{-j} b_{j} .
\end{array}\right\}
$$

We define

$$
\kappa(t)=\kappa_{0}(t) \mathbf{1}_{\left[t_{0},+\infty[\right.}(t)+\sum_{j=1}^{\infty} \kappa_{j}(t) \mathbf{1}_{\left[t_{j}, t j-1[\right.}(t),
$$

observing that $t_{j}<b_{j} / 2^{j}<b_{0} / 2^{j} \rightarrow 0$ as $j \rightarrow \infty$, so by conditions (3.6), $\kappa$ is a strictly increasing convex map defined on $] 0,+\infty[$. The convexity follows from the continuity and from the fact that the sequence of slopes $\left(b_{j}\right)$ decreases as the intervals get close to the origin. By the construction of $\kappa$ we have that

$$
\begin{aligned}
& \liminf _{t \rightarrow 0_{+}} \frac{\kappa(t)}{t^{2}} \leqslant \limsup _{j \rightarrow \infty} \frac{\kappa\left(t_{j}\right)}{t_{j}^{2}} \leqslant \lim _{j \rightarrow \infty} \varepsilon_{j}=0, \\
& \limsup _{t \rightarrow 0_{+}} \frac{\kappa(t)}{t^{2}} \geqslant \liminf _{j \rightarrow \infty} \frac{\kappa\left(\tau_{j}\right)}{\tau_{j}^{2}} \geqslant \lim _{j \rightarrow \infty} m_{j}=+\infty .
\end{aligned}
$$

The sequence $\left(\kappa\left(t_{j}\right)\right)$ converges to zero as $j \rightarrow \infty$ and $\kappa$ is monotone, so $\kappa(t) \rightarrow 0$ as $t \rightarrow 0_{+}$and $\kappa$ is continuous at the origin. Thus, we have proved the existence of a strictly increasing convex map $\kappa:[0,+\infty[\rightarrow[0,+\infty[$ which is continuous at the origin with $\kappa(0)=0$ and which satisfies (3.7) and (3.8). These two conditions are of course just (3.4), so our proof is finished.

Acknowledgements. V.M. is grateful to Bruce Kleiner for a stimulating conversation on the metric differentiability problem and also thanks the Max-Plank-Institut in Leipzig for kind hospitality during this collaboration.

\section{References}

1. L. Ambrosio And B. Kirchheim, Rectifiable sets in metric and Banach spaces, Math. Ann. 318 (2000), 527-555.

2. Z. M. BALOGH, Size of characteristic sets and functions with prescribed gradients, $J$. Reine Angew. Math., in press.

3. G. David And S. Semmes, Fractured fractals and broken dreams. Self-similar geometry through metric and measure, Oxford Lecture Series in Mathematics and its Applications, vol. 7 (Oxford University Press, 1997).

4. H. FEDERER, Geometric measure theory (Springer, 1969). 
5. G. B. Folland And E. M. Stein, Hardy spaces on homogeneous groups (Princeton University Press, Princeton, NJ, 1982).

6. B. Franchi, R. Serapioni and F. Serra Cassano, Rectifiability and perimeter in the Heisenberg group, Math. Ann. 321 (2001), 479-531.

7. B. Franchi, R. Serapioni And F. Serra Cassano, Regular hypersurfaces, intrinsic perimeter and implicit function theorem in Carnot groups, Commun. Analysis Geom., in press.

8. J. Heinonen And S. Semmes, Thirty-three yes or no questions about mappings, measures, and metrics, Conform. Geom. Dyn. 1 (1997), 1-12.

9. B. KirchHeim, Rectifiable metric spaces: local structure and regularity of the Hausdorff measure, Proc. Am. Math. Soc. 121 (1994), 113-123.

10. N. J. KorevaAr And R. M. Schoen, Sobolev spaces and harmonic maps for metric space targets, Commun. Analysis Geom. 1 (1993), 561-659.

11. V. Magnani, Differentiability and area formula on stratified Lie groups, Houston J. Math. 27 (2001), 297-323.

12. V. Magnani, On a general coarea inequality and applications, Ann. Acad. Sci. Fenn. Math. 27 (2002), 121-140.

13. R. Monti and F. Serra Cassano, Surface measures in Carnot-Carathédory spaces, Calc. Var. 13 (2001), 339-376.

14. P. Mattila, Geometry of sets and measures in Euclidean spaces (Cambridge University Press, 1995).

15. P. PAnsu, Métriques de Carnot-Carathéodory et quasiisométries des espaces symétriques de rang un, Ann. Math. (2) 129 (1989), 1-60.

16. S. D. PAuls, The large scale geometry of nilpotent Lie groups, Commun. Analysis Geom. 9 (2001), 951-982.

17. S. D. PAUls, A notion of rectifiability modelled on Carnot groups, preprint.

18. S. K. VOdOP'YANOV, $\mathcal{P}$-differentiability on Carnot groups in different topologies and related topics, in Proc. Analysis and Geometry, pp. 603-670 (Sobolev Institute Press, Novosibirsk, 2000). 